\title{
An Orientation Theorem with Parity Conditions
}

\author{
András Frank* $^{*}$ Tibor Jordán ${ }^{\dagger} \quad$ Zoltán Szigeti $^{\ddagger}$ \\ Final version, January 18, 2000
}

\begin{abstract}
Given a graph $G=(V, E)$ and a set $T \subseteq V$, an orientation of $G$ is called $T$-odd if precisely the vertices of $T$ get odd in-degree. We give a good characterization for the existence of a $T$-odd orientation for which there exist $k$ edge-disjoint spanning arborescences rooted at a prespecified set of $k$ roots. Our result implies Nash-Williams' theorem on covering the edges of a graph by $k$ forests and a (generalization of a) theorem due to Nebeský on upper embeddable graphs.
\end{abstract}

\section{Introduction}

Let $G=(V, E)$ be a graph. We denote the number of components of $G$ by $c(G)$ and we use $\beta(G)$ to denote $|E(G)|-|V(G)|+c(G)$. Let $G$ be a connected graph. An embedding of $G$ into an orientable surface is called a 2-cell embedding if every region is homeomorphic to an open disk. The maximum integer $g$ for which there exists a 2-cell embedding of $G$ into an orientable surface of genus $g$ is the maximum genus of $G$, denoted by $\gamma_{M}(G)$. It is known [?] that $\gamma_{M}(G) \leq\lfloor\beta(G) / 2\rfloor$. Graphs with $\gamma_{M}(G)=\lfloor\beta(G) / 2\rfloor$ are called upper embeddable.

The following theorem of Xuong gave the first characterization of maximum genus and was used later by Furst et al. [?] to construct a polynomial-time algorithm to compute $\gamma_{M}(G)$. For a spanning tree $F$ of $G$ let $x_{G}(F)$ denote the number of those components $C$ of $G-E(F)$ for which $|E(C)|$ is odd. Let $\mathcal{F}(G)$ denote the set of spanning trees of $G$.

Theorem 1.1 [?] If $G$ is a connected graph then

$$
\gamma_{M}(G)=\left(\beta(G)-\min \left\{x_{G}(F): F \in \mathcal{F}(G)\right\}\right) / 2 .
$$

*Department of Operations Research, Eötvös University, H-1088 Rákóczi út 5., Budapest, Hungary, and Ericsson Traffic Laboratory, H-1037 Laborc u. 1, Budapest, Hungary. e-mail: frank@cs . elte. hu

${ }^{\dagger}$ BRICS, Department of Computer Science, University of Aarhus, Ny Munkegade, building 540, DK-8000 Aarhus, Denmark. e-mail: jordan@daimi.au.dk

${ }^{\ddagger}$ Equipe Combinatoire, Université Paris VI, 4, place Jussieu, 75252 Paris, France. e-mail: szigeti@ecp6.jussieu.fr 
Notice that $\beta(G)-x_{G}(F)$ is even for every spanning tree $F$. A spanning tree $F$ of $G$ is called an even tree of $G$ if each connected component of $G-E(F)$ has an even number of edges. A special case of Theorem ?? (see also Jungerman [?]) is the following.

Corollary 1.2 Let $G$ be a connected graph with even $\beta(G)$. Then $G$ is upper embeddable if and only if it has an even tree.

Some years later Nebeský found the first NP $\cap$ co-NP characterization of $\gamma_{M}(G)$ by proving the following equality.

Theorem 1.3 [?] A connected graph $G=(V, E)$ satisfies

$$
\min \left\{x_{G}(F): F \in \mathcal{F}(G)\right\}=\max \{c(G-A)+b(G-A)-|A|-1: A \subseteq E\},
$$

where $b(G-A)$ denotes the number of those components $D$ of $G-A$ for which $\beta(D)$ is odd.

Specializing this result to upper embeddable graphs $G$ of even $\beta(G)$ gives:

Theorem 1.4 [?] A connected graph $G=(V, E)$ has an even tree if and only if

$$
|A| \geq c(G-A)+b(G-A)-1
$$

holds for every $A \subseteq E$.

For more results on maximum genus see the survey paper [?]. The starting point of our investigations is the purely graph theoretic Theorem ?? which involves parity as well as connectivity conditions. We shall prove (generalizations of) this result from a new viewpoint (namely, in terms of orientations of $G$ ) and hence give a new framework which links this result to other, probably more familiar results in graph theory.

Theorem ?? can be reformulated in terms of orientations of $G$. An orientation of an undirected graph $G$ is a directed graph obtained from $G$ by assigning an orientation to each edge of $G$. A subset $F$ of edges of a directed graph is a spanning arborescence rooted at vertex $r$ if $F$ forms a spanning tree in the underlying undirected graph and each vertex has in-degree one in $F$, except the root $r$.

Let $G=(V, E)$ be a connected undirected graph and let $T \subseteq V$. An orientation of $G$ is called $T$-odd if precisely the vertices of $T$ get odd in-degree. It is easy to see that $G$ has a $T$-odd orientation if and only if $|E|+|T|$ is even. (This can be seen by induction as follows. Take $v \in V$ for which $G-v$ is connected. If $v \in T$ and $d(v)$ is odd or $v \notin T$ and $d(v)$ is even then orient the edges incident to $v$ towards $v$ and apply induction on $G-v$ with respect to $T-v$ to obtain the orientation of the rest. If this is not the case then orient all edges but one, say $v x$, towards $v$, and apply induction on $G-v$ with respect to $(T-v) \oplus\{x\}$. Here $\oplus$ denotes the symmetric difference.) ¿From this fact we obtain the following observation (which was found earlier by Chevalier et al. [?], too). 
Proposition 1.5 Let $G=(V, E)$ be a connected graph for which $\beta(G)$ is even and let $r \in V$. Then $G$ has an even tree if and only if there exists a $(V-r)$-odd orientation of $G$ which contains a spanning arborescence rooted at $r$.

Corollary 1.6 Let $G=(V, E)$ be a connected graph for which $\beta(G)$ is even and let $r \in V$. Then $G$ has a $(V-r)$-odd orientation which contains a spanning arborescence rooted at $r$ if and only if (??) holds for every $A \subseteq E$.

Motivated by Theorem ?? and Corollary ??, we investigate more general problems concerning orientations of undirected graphs simultaneously satisfying connectivity and parity requirements. Namely, given an undirected graph $G=(V, E), T \subseteq V$ and $k \geq 0$, our main result gives a necessary and sufficient condition for the existence of a $T$-odd orientation of $G$ which contains $k$ edge-disjoint spanning arborescences rooted at a given set of $k$ roots. This good characterization generalizes Theorem ?? and at the same time slightly simplifies condition (??). Furthermore, it implies Nash-Williams' theorem on covering the edges of a graph by $k$ forests as well. We also point out some connections to a related problem on $k$-edge-connected $T$-odd orientations which was investigated in [?]. These corollaries are discussed in Section 3.

The proof of our main result employs the proof method which was developed independently by Gallai and Anderson and which was first used to show an elegant proof for Tutte's theorem on perfect matchings of graphs, see [?]. In our case the weaker result the proof hinges on (which is Hall's theorem in the previously mentioned proof for Tutte's result) is an orientation theorem of the first author (Theorem ?? below).

Let $R=\left\{r_{1}, \ldots, r_{k}\right\}$ be a multiset of vertices of $G$ (that is, the elements of $R$ are not necessarily pairwise distinct). By $T \oplus R$ we mean $\left(\left(T \oplus r_{1}\right) \oplus \ldots\right) \oplus r_{k}$. For some $X \subseteq V$ the subgraph induced by $X$ is denoted by $G[X]$. The number of edges in $G[X]$ is denoted by $i(X)$. For a partition $\mathcal{P}=\left\{V_{1}, \ldots, V_{t}\right\}$ of $V$ with $t$ elements the set of edges connecting different elements of $\mathcal{P}$ is denoted by $E(\mathcal{P})$. We set $e(\mathcal{P})=|E(\mathcal{P})|$. The in-degree of a set $X \subseteq V$ in a directed graph $D=(V, E)$ is denoted by $\rho(X)$. The following well-known result is due to Edmonds.

Theorem 1.7 [?] Let $R$ be a multiset of vertices of size $k$ in a directed graph $D=(V, E)$. Then $D$ contains $k$ edge-disjoint spanning arborescences rooted at $R$ if and only if

$$
\rho(X) \geq k-|X \cap R| \text { for every } X \subseteq V .
$$

The following result is due to Frank.

Theorem 1.8 [?, Theorem 2.1] Let $H=(V, E)$ be a graph, and let $g: V \rightarrow Z_{+}$be a function. Then there exists an orientation of $H$ whose in-degree function $\rho$ satisfies $\rho(v)=g(v)$ for every $v \in V$ if and only if the following two conditions hold.

$$
\begin{gathered}
g(V)=|E| \\
g(X) \geq i(X) \text { for every } \emptyset \neq X \subseteq V .
\end{gathered}
$$


We shall rely on the following orientation theorem, which is easy to prove from Theorem ?? and Theorem ??.

Theorem 1.9 Let $H=(V, E)$ be a graph, let $R=\left\{r_{1}, \ldots, r_{k}\right\}$ be a multiset of $k$ vertices of $H$ and let $g: V \rightarrow Z_{+}$be a function. Then there exists an orientation of $H$ whose in-degree function $\rho$ satisfies $\rho(v)=g(v)$ for every $v \in V$ and for which there exist $k$ edge-disjoint spanning arborescences with roots $\left\{r_{1}, \ldots, r_{k}\right\}$ if and only if (??) and the following condition hold.

$$
g(X) \geq i(X)+k-|X \cap R| \text { for every } \emptyset \neq X \subseteq V
$$

Proof: It is easy to see that both (??) and (??) are necessary. To see the other direction let us take an orientation of $H$ whose in-degree function $\rho$ satisfies $\rho(v)=g(v)$ for every $v \in V$. By (??) and since (??) implies (??), such an orientation exists by Theorem ??. We claim that this directed graph satisfies (??) with respect to the multiset $R$ of roots. Indeed, by observing that $\rho(X)=\sum_{x \in X} \rho(x)-i(X)=\sum_{x \in X} g(x)-i(X)=g(X)-i(X)$ for every $X \subseteq V$, by (??) we obtain $\rho(X) \geq i(X)+k-|X \cap R|-i(X)=k-|X \cap R|$, as required. Hence we are done by Theorem ??.

Note that $R$ is a multiset in Theorem ??, hence by $|X \cap R|$ in (??) we mean $\mid\left\{r_{i} \in R: r_{i} \in\right.$ $X, i=1, \ldots, k\} \mid$. This convention will be used later on, whenever we take the intersection (or union) with a multiset.

Given $G=(V, E), T \subseteq V, k \in Z_{+}$and a partition $\mathcal{P}=\left\{V_{1}, \ldots, V_{t}\right\}$ of $V$, an element $V_{j}$ $(1 \leq j \leq t)$ is called odd if $\left|V_{j} \cap T\right|-i\left(V_{j}\right)-k$ is odd, otherwise $V_{j}$ is even. The number of odd elements of $\mathcal{P}$ is denoted by $s_{G}(\mathcal{P}, T, k)$ (where some parameters may be omitted if they are clear from the context). Our main result is the following.

Theorem 1.10 Let $G=(V, E)$ be a graph, $T \subseteq V$ and let $k \geq 0$ be an integer. For a multiset of $k$ vertices $R=\left\{r_{1}, \ldots, r_{k}\right\}$ of $V$ there exists a $T \oplus R$-odd orientation of $G$ for which there exist $k$ edge-disjoint spanning arborescences with roots $\left\{r_{1}, \ldots, r_{k}\right\}$ if and only if

$$
e(\mathcal{P}) \geq k(t-1)+s(\mathcal{P}, T)
$$

holds for every partition $\mathcal{P}=\left\{V_{1}, \ldots V_{t}\right\}$ of $V$.

Note that taking $\mathcal{P}=\{V\}$ in (??) yields the condition $|T|-|E|-k$ is even. Also notice that the multiset $R$ of roots plays no role in the characterization. This fact is easy to see directly by observing that if $G$ has a proper $T \oplus R$-odd orientation for some $R$ then $G$ has a proper $T \oplus R^{\prime}$-odd orientation for any other multiset $R^{\prime}$ of $k$ roots. Indeed, by repeatedly taking two roots $r \in R-R^{\prime}$ and $r^{\prime} \in R^{\prime}-R$ and a spanning arborescence $F$ of root $r$ in the proper $T \oplus R$ odd orientation and then reversing the edges of the directed $r-r^{\prime}$ path in $F$, one obtains a proper $T \oplus R^{\prime}$-odd orientation. In the proof, however, the most general formulation will be convenient. 


\section{The Proof of Theorem ??}

Proof: (of Theorem ??) To see the necessity of condition (??), consider an orientation of $G$ with the required properties and some partition $\mathcal{P}=\left\{V_{1}, \ldots, V_{t}\right\}$ of $V$. The following fact is easy to observe.

Proposition 2.1 For every $T \oplus R$-odd orientation of $G$ and for every $V_{j}(1 \leq j \leq t)$ we have $\left|V_{j} \cap T\right|-i\left(V_{j}\right)-k \equiv \rho\left(V_{j}\right)-\left(k-\left|V_{j} \cap R\right|\right) \quad(\bmod 2)$.

Proof: Since the orientation is $T \oplus R$-odd, we obtain $\rho\left(V_{j}\right)+i\left(V_{j}\right)=\sum_{v \in V_{j}} \rho(v) \equiv \mid V_{j} \cap(T \oplus$ $R)|\equiv| V_{j} \cap T|-| V_{j} \cap R \mid$ and the claim follows.

Since there exist $k$ edge-disjoint arborescences rooted at vertices of $R$, it follows from the easy part of Theorem ?? that $\rho\left(V_{j}\right)-\left(k-\left|V_{j} \cap R\right|\right) \geq 0$ for each $V_{j}$. If this number is odd (or, equivalently by Proposition ??, if $V_{j}$ is odd) then it is at least one. This yields $e(\mathcal{P})=$ $\sum_{V_{j} \in \mathcal{P}} \rho\left(V_{j}\right)=\sum_{V_{j} \in \mathcal{P}}\left(\rho\left(V_{j}\right)-\left(k-\left|V_{j} \cap R\right|\right)\right)+k t-|V \cap R| \geq s(\mathcal{P}, T)+k t-k=$ $k(t-1)+s(\mathcal{P}, T)$, hence the necessity follows.

In what follows we prove that (??) is sufficient. An orientation is called good if the directed graph obtained contains $k$ edge disjoint spanning arborescences rooted at $R$. Let us suppose that the statement of the theorem does not hold and let us take a counter-example (that is, a graph $G=(V, E)$ with $T, R$ and $k$, for which (??) holds but no good $T \oplus R$-odd orientation exists) for which $|V|+|E|$ is as small as possible.

Proposition $2.2 e(\mathcal{P}) \equiv k(t-1)+s(\mathcal{P}, T) \quad(\bmod 2)$ for every partition $\mathcal{P}$.

Proof: By choosing $\mathcal{P}_{0}=\{V\}$ in (??) we obtain that $|T|-|E|-k$ is even. This implies $s(\mathcal{P}, T) \equiv \sum_{1}^{t}\left(\left|V_{j} \cap T\right|-i\left(V_{j}\right)-k\right)=|T|-(|E|-e(\mathcal{P}))-k t=|T|-|E|-k+e(\mathcal{P})-k(t-1) \equiv$ $e(\mathcal{P})-k(t-1)$.

We call a partition $\mathcal{P}$ of $V$ consisting of $t$ elements tight if $e(\mathcal{P})=k(t-1)+s(\mathcal{P}, T)$ and $t \geq 2$.

Lemma 2.3 There exists a tight partition of $V$.

Proof: Let $a b$ be an arbitrary edge of $G$. Focus on the graph $G^{\prime}=G-a b$ and the modified set $T^{\prime}=T \oplus b$. If there was a good $T^{\prime} \oplus R$-odd orientation of $G^{\prime}$ then adding the arc $a b$ would provide a good $T \oplus R$-odd orientation of $G$, which is impossible. Thus, by the minimality of $G$, there exists a partition $\mathcal{P}$ of $V$ consisting of $t$ elements violating (??) in $G^{\prime}$, that is, by Proposition ??, $e_{G^{\prime}}(\mathcal{P})+2 \leq k(t-1)+s_{G^{\prime}}\left(\mathcal{P}, T^{\prime}\right)$. Clearly, $t \geq 2$ holds.

For the same partition in $G$ we have $e_{G}(\mathcal{P}) \leq e_{G^{\prime}}(\mathcal{P})+1$ and also $s_{G}(\mathcal{P}, T) \geq s_{G^{\prime}}\left(\mathcal{P}, T^{\prime}\right)-1$, since adding the edge $a b$ and replacing $T \oplus b$ by $T$ may change the parity of at most one element of the partition. Thus $k(t-1)+s_{G}(\mathcal{P}, T) \geq k(t-1)+s_{G^{\prime}}\left(\mathcal{P}, T^{\prime}\right)-1 \geq e_{G^{\prime}}(\mathcal{P})+2-1 \geq$ 
$e_{G}(\mathcal{P}) \geq k(t-1)+s_{G}(\mathcal{P}, T)$, hence $\mathcal{P}$ is tight in $G$ and the lemma follows.

Let us fix a tight partition $\mathcal{P}=\left\{V_{1}, \ldots, V_{t}\right\}$ in $G$ for which $t$ is maximal. Denote the number of odd components of $\mathcal{P}$ by $s$.

Lemma 2.4 Every element $V_{j}$ of $\mathcal{P}(1 \leq j \leq t)$ has the following property:

(a) if $V_{j}$ is even, then (??) holds in $G\left[V_{j}\right]$, with respect to $T \cap V_{j}$,

(b) if $V_{j}$ is odd, then for each vertex $v \in V_{j}$, (??) holds in $G\left[V_{j}\right]$, with respect to $\left(T \cap V_{j}\right) \oplus v$.

Proof: We handle the two cases simultaneously. Suppose that there exists a partition $\mathcal{P}^{\prime}$ of $t^{\prime}$ elements in $G\left[V_{j}\right]$ violating (??) (with respect to $T \cap V_{j}$ in case (a) or with respect to $\left(T \cap V_{j}\right) \oplus v$, for some $v \in V_{j}$, in case (b)). By Proposition ?? this implies $k\left(t^{\prime}-1\right)+s^{\prime} \geq e\left(\mathcal{P}^{\prime}\right)+2$, where $s^{\prime}$ denotes the number of odd elements of $\mathcal{P}^{\prime}$. Consider the partition $\mathcal{P}^{\prime \prime}=\left(\mathcal{P}-V_{j}\right) \cup \mathcal{P}^{\prime}$, consisting of $t^{\prime \prime}$ elements from which $s^{\prime \prime}$ are odd. Clearly, $e\left(\mathcal{P}^{\prime \prime}\right)=e(\mathcal{P})+e\left(\mathcal{P}^{\prime}\right)$ and $t^{\prime \prime}=t+t^{\prime}-1$. Furthermore, $s^{\prime \prime} \geq s+s^{\prime}-2$, since the parity of at most two elements may be changed (these are $V_{j}$ - only in case (b) - and the element in $\mathcal{P}^{\prime}$ which contains the vertex $v$ - only in case (b) again). Since (??) holds for $\mathcal{P}^{\prime \prime}$ by the assumption of the theorem, we have $k\left(t^{\prime \prime}-1\right)+s^{\prime \prime} \leq e\left(\mathcal{P}^{\prime \prime}\right)=$ $e(\mathcal{P})+e\left(\mathcal{P}^{\prime}\right) \leq k(t-1)+s+k\left(t^{\prime}-1\right)+s^{\prime}-2=k\left(\left(t+t^{\prime}-1\right)-1\right)+s+s^{\prime}-2 \leq k\left(t^{\prime \prime}-1\right)+s^{\prime \prime}$. Thus $\mathcal{P}^{\prime \prime}$ is a tight partition with $t^{\prime \prime}>t$, which contradicts the choice of $\mathcal{P}$.

Let us denote the graph obtained from $G$ by contracting each element $V_{j}$ of $\mathcal{P}$ into a single vertex $v_{j}(1 \leq j \leq t)$ by $H$. Let $R^{\prime}=\left\{r_{1}^{\prime}, \ldots, r_{k}^{\prime}\right\}$ denote the multiset of vertices of $H$ corresponding to the vertices of $R$ in $G$ (that is, every root in some $V_{j}$ yields a new root $v_{j}$ ). Furthermore, let $A$ denote those vertices of $H$ which correspond to odd elements of $\mathcal{P}$ and let $B=V(H)-A$. Note that since $\mathcal{P}$ is tight, we have

$$
|E(H)|=e(\mathcal{P})=k(t-1)+s(\mathcal{P}) .
$$

Now define the following function $g$ on the vertex set of $H$.

$$
g\left(v_{j}\right)= \begin{cases}k+1-\left|V_{j} \cap R\right| & \text { if } v_{j} \in A \\ k-\left|V_{j} \cap R\right| & \text { otherwise. }\end{cases}
$$

Lemma 2.5 There exists an orientation of $H$ whose in-degree function is $g$ and which contains $k$ edge-disjoint spanning arborescences with roots $\left\{r_{1}^{\prime}, \ldots, r_{k}^{\prime}\right\}$.

Proof: To prove the lemma we have to verify that the two conditions (??) and (??) of Theorem ?? are satisfied. First we can see that $g(V(H))=g(A)+g(B)=s(k+1)+(t-s) k-k=$ $k(t-1)+s=|E(H)|$ by the definition of $g$ and by (??). Thus (??) is satisfied.

To verify (??), let us choose an arbitrary non-empty subset $X$ of $V(H)$. Let us define the partition $\mathcal{P}^{*}$ of $V(G)$ by $\mathcal{P}^{*}:=\left\{V_{j}: v_{j} \in V(H)-X\right\} \cup \bigcup_{v_{j} \in X} V_{j}$. Then $\mathcal{P}^{*}$ has $t^{*}=t-|X|+1$ elements and the number of its odd elements $s^{*}$ is at least $s-|X \cap A|$. Applying (??) for $\mathcal{P}^{*}$, it follows that $k\left(t^{*}-1\right)+s^{*} \leq e\left(\mathcal{P}^{*}\right)$. Hence $k((t-|X|+1)-1)+s-|X \cap A| \leq k\left(t^{*}-1\right)+s^{*} \leq$ 
$e\left(\mathcal{P}^{*}\right)=e(\mathcal{P})-i(X)=k(t-1)+s-i(X)$. From this it follows that $i(X)+k \leq k|X|+|X \cap A|$. Therefore $i(X)+k-\left|X \cap R^{\prime}\right| \leq k|X|+|X \cap A|-\left|X \cap R^{\prime}\right|=|X \cap A|(k+1)+|X \cap B| k-\left|X \cap R^{\prime}\right|=$ $g(X \cap A)+g(X \cap B)=g(X)$, proving that (??) is also satisfied.

Let us fix an orientation of $H$ whose in-degree function $\rho_{H}=g$ and which contains a set $\mathcal{F}$ of $k$ edge-disjoint spanning arborescences $\left\{F_{1}, \ldots, F_{k}\right\}$ with roots $\left\{r_{1}^{\prime}, \ldots, r_{k}^{\prime}\right\}$. Such an orientation exists by Lemma ??. Observe, that this orientation of $H$ corresponds to a partial orientation of $G$ (namely, an orientation of the edges of $E(\mathcal{P})$ ).

For any vertex $v_{j}$ of $H$ there are $g\left(v_{j}\right)$ arcs entering $v_{j}$. If $V_{j}$ is even then each arc entering $v_{j}$ belongs to some arborescence in $\mathcal{F}$. If $V_{j}$ is odd then each arc entering $v_{j}$ except exactly one belongs to some arborescence of $\mathcal{F}$, by the definition of $g$.

For an arbitrary $V_{j} \in \mathcal{P}$ let us denote by $R_{j}^{H}$ the multiset of those vertices in $V_{j}$ which are the heads of the arcs of this partial orientation entering $V_{j}$ and belonging to some arborescence in $\mathcal{F}$. By the definition of $g$, we have $\left|R_{j}^{H}\right|=k-\left|V_{j} \cap R\right|$. Let $R_{j}=\left(V_{j} \cap R\right) \cup R_{j}^{H}$. Note that $\left|R_{j}\right|=\left|V_{j} \cap R\right|+\left|R_{j}^{H}\right|=k$. Furthermore, if $V_{j}$ is odd then let us denote by $a_{j}$ the vertex in $V_{j}$ which is the head of the unique arc entering $V_{j}$ and not belonging to any arborescence in $\mathcal{F}$. Let $T_{j}=T \cap V_{j}$ if $V_{j}$ is even and let $T_{j}=\left(T \cap V_{j}\right) \oplus a_{j}$ if $V_{j}$ is odd.

By the minimality of $G$ and since $\left|V_{j}\right|<|V(G)|$ for each $1 \leq j \leq t$, Lemma ?? implies that for each $j$ there exists a $T_{j} \oplus R_{j}$-odd orientation of $G\left[V_{j}\right]$ which contains $k$ edge-disjoint spanning arborescences with roots in $R_{j}$. Combining these orientations of the subgraphs induced by the elements of $\mathcal{P}$ and the orientation of $E(\mathcal{P})$ obtained earlier, we get an orientation of $G$. This orientation is clearly a good $T \oplus R$-odd orientation of $G$, contradicting our assumption on $G$. This contradiction proves the theorem.

\section{Corollaries}

As we reformulated Theorem ?? in terms of odd orientations and spanning arborescences in Corollary ??, we can similarly reformulate Theorem ?? in terms of even components and spanning trees.

Theorem 3.1 A graph $G=(V, E)$ has $k$ edge-disjoint spanning trees $F_{1}, \ldots, F_{k}$ so that each connected component of $G-\cup_{1}^{k} E\left(F_{i}\right)$ has an even number of edges if and only if

$$
e(\mathcal{P}) \geq k(t-1)+s
$$

holds for each partition $\mathcal{P}=\left\{V_{1}, \ldots V_{t}\right\}$ of $V$, where $s$ is the number of those elements of $\mathcal{P}$ for which $i\left(V_{j}\right)+k\left(\left|V_{j}\right|-1\right)$ is odd.

Proof: As we observed, $G$ has an oriention for which the in-degree of every vertex is even if and only if each connected component of $G$ contains an even number of edges. Thus the desired spanning trees exist in $G$ if and only if $G$ has a $T \oplus R$-odd orientation which contains $k$ edgedisjoint $r$-arborescences, where $T=V$, if $k$ is odd, $T=\emptyset$, if $k$ is even, and $R=\left\{r_{1}, \ldots, r_{k}\right\}$, 
$r_{i}=r(i=1, \ldots, k)$ for an arbitrary $r \in V$. Based on this fact, Theorem ?? proves the theorem (by observing that (??) specializes to (??) due to the special choice of $T$ ).

The special case $k=1$ of Theorem ?? corresponds to Theorem ??. Since (??) implies (??) if $k=1$, Theorem ?? applies and we obtain a slightly simplified version of Nebeský's result. Note also that our main result provides a proof of different nature for Theorem ?? by using Theorem ??.

The next corollary we prove is Nash-Williams' classical theorem on forest covers.

Corollary 3.2 [?] The edges of a graph $G=(V, E)$ can be covered by $k$ forests if and only if

$$
i(X) \leq k(|X|-1)
$$

holds for every $\emptyset \neq X \subseteq V$.

Proof: We consider the sufficiency of the condition. Let $G=(V, E)$ be a graph for which (??) holds. The first claim is that we can add new edges to $G$ until the number of edges equals $k(|V|-1)$ without destroying (??). To see this, observe that the addition of a new edge $e=x y$ (which may be parallel to some other edges already present in $G$ ) cannot be added if and only if $x, y \in Z$ for some $Z \subseteq V$ with $i(Z)=k(|Z|-1)$. Such a set, satisfying (??) with equality, will be called full. It is well-known that the function $i: 2^{V} \rightarrow Z_{+}$is supermodular, that is, it satisfies $i(X)+i(Y) \leq i(X \cap Y)+i(X \cup Y)$ for every $X, Y \subseteq V$. Therefore for two intersecting full sets $Z$ and $W$ we have $k(|Z|-1)+k(|W|-1)=i(Z)+i(W) \leq i(Z \cap W)+i(Z \cup W) \leq$ $k(|Z \cap W|-1)+k(|Z \cup W|-1)=k(|Z|-1)+k(|W|-1)$. Thus equality holds everywhere, and the sets $Z \cap W$ and $Z \cup W$ are also full. Now let $F$ be a maximal full set (we may assume $F \neq V$ ) and $e=x y$ for some pair $x \in F, y \in V-F$. If we destroyed (??) by adding $e$, we would have a full set $x, y \in F^{\prime}$ in $G$ intersecting $F$, hence $F \cup F^{\prime}$ would also be full by our previous observation. This contradicts the maximality of $F$.

Thus in the rest of the proof we may assume that $|E|=k(|V|-1)$. We claim that there exist $k$ edge-disjoint spanning trees in $G$. The existence of these trees immediately implies that $G$ can be covered by $k$ forests because $|E|=k(|V|-1)$.

By Theorem ??, it is enough to prove that (??) holds in $G$. Let $\mathcal{P}=\left\{V_{1}, \ldots, V_{t}\right\}$ be a partition of $V$ and let $V_{1}, \ldots, V_{s}$ denote the odd elements of $\mathcal{P}$ (with respect to $k$ ). Observe that for an odd element $V_{j}$ the parity of $i\left(V_{j}\right)$ and $k\left(\left|V_{j}\right|-1\right)$ must be different (this holds for even $k$ and for odd $k$ as well), hence these numbers cannot be equal. Thus we can count as follows: $e(\mathcal{P})=|E|-\sum i\left(V_{i}\right)=k(|V|-1)-\sum\left(i\left(V_{i}\right): V_{i}\right.$ is even $)-\sum\left(i\left(V_{j}\right)\right.$ : $V_{j}$ is odd $) \geq k(|V|-1)-\sum\left(k\left(\left|V_{i}\right|-1\right): V_{i}\right.$ is even $)-\sum\left(k\left(\left|V_{j}\right|-1\right)-1: V_{j}\right.$ is odd $)=$ $k(|V|-1)-k|V|+k t+s=k(t-1)+s$, as required.

Finally we point out some connections to a related problem which was solved recently by Frank and Király [?]. In an undirected graph $G=(V, E)$ a set $T \subseteq V$ is $G$-even if $|T|+|E|$ is even. As we remarked, $G$ has a $T$-odd orientation if and only if $T$ is $G$-even. Given a graph $G$ and a $G$-even subset $T \subseteq V(G)$ one may ask for a necessary and sufficient condition for the 
existence of a $T$-odd orientation of $G$ which is $k$-edge-connected. (A directed graph $G=(V, E)$ is $k$-edge-connected if $\rho(X) \geq k$ holds for every $\emptyset \neq X \subset V$, where $\rho(X)$ denotes the number of edges entering $X$.) This question is still open, even for $k=1$. On the other hand, Frank and Király characterized those graphs $G$ which have $k$-edge-connected $T$-odd orientations for every $G$-even $T$.

One of their main results is as follows:

Theorem 3.3 [?] Let $G$ be an undirected graph and $k$ be a positive integer. Then $G$ has a $k$-edge-connected $T$-odd orientation for every $G$-even subset $T \subseteq V$ if and only if

$$
e(\mathcal{P}) \geq(k+1) t-1
$$

holds for every partition $\mathcal{P}=\left\{V_{1}, \ldots V_{t}\right\}, t \geq 2$, of $V$.

Our results imply a similar condition for those graphs $G$ which have a $T \oplus R$-odd orientation with $k$ edge-disjoint spanning arborescences rooted at $R$ for every $T$ and every set $R$ of $k$ roots.

Theorem 3.4 Let $G=(V, E)$ be an undirected graph and $k$ be a positive integer. Then $G$ has a $T \oplus R$-odd orientation for which there exist $k$ edge-disjoint spanning arborescences with roots $\left\{r_{1}, \ldots, r_{k}\right\}$ for every subset $T \subseteq V$ with $|T|+|E|-k$ even (and for an arbitrarily chosen multiset $R=\left\{r_{1}, \ldots, r_{k}\right\} \subseteq V$ of $k$ roots) if and only if

$$
e(\mathcal{P}) \geq(k+1)(t-1)
$$

holds for every partition $\mathcal{P}=\left\{V_{1}, \ldots V_{t}\right\}, t \geq 2$, of $V$.

Proof: First suppose $G$ satisfies (??) and take some $T \subseteq V$ with $|T|+|E|-k$ even. Clearly, $s(\mathcal{P}, T) \leq t$ for any partition $\mathcal{P}$ consisting of $t$ classes. Thus $e(\mathcal{P}) \geq(k+1)(t-1)=k(t-1)+$ $t-1$ shows that $G$ satisfies (??) for every partition $\mathcal{P}$ with $t \geq 2$ unless $e(\mathcal{P})=(k+1)(t-1)$ and $s(\mathcal{P}, T)=t$. However, by Proposition ?? every partition $\mathcal{P}^{\prime}$ satisfies $e\left(\mathcal{P}^{\prime}\right) \equiv k(t-1)+s\left(\mathcal{P}^{\prime}, T\right)$ $(\bmod 2)$ and hence $(k+1)(t-1) \equiv k(t-1)+s(\mathcal{P}, T) \quad(\bmod 2)$ follows. Thus the latter case cannot occur. This shows (??) holds for every $T$ and every $\mathcal{P}$. Therefore Theorem ?? implies that $G$ has the desired $T \oplus R$-odd orientation for $T$ and for every set $R$ of $k$ roots.

Conversely, suppose that for some partition $\mathcal{P}=\left\{V_{1}, \ldots V_{t}\right\}, t \geq 2$, we have $e(\mathcal{P})<(k+$ 1) $(t-1)$. Now by defining $T \subseteq V$ appropriately, we can make at least $t-1$ classes $V_{i}$ "odd" and hence $s(\mathcal{P}, T) \geq t-1$. (Since $|T|+|E|-k$ has to be even, the parity of $s(\mathcal{P}, T)$ is determined.) Thus for this $T$ and $\mathcal{P}$ we have $e(\mathcal{P})<(k+1)(t-1)=k(t-1)+t-1 \leq k(t-1)+s(\mathcal{P}, T)$, showing that $G$ violates (??). Thus, by the easy part of Theorem ??, the required orientation does not exist for every $T$ in $G$.

Notice that by a theorem of Tutte and Nash-Williams on edge-disjoint spanning trees, condition (??) is equivalent to the fact that $G$ has $k+1$ edge-disjoint spanning trees. Using this, one can obtain a different proof for Theorem ??. 


\section{Remarks}

The problem of finding a spanning tree of Theorem ?? with the required properties (or more generally, the problem of computing $\min \left\{x_{G}(F): F \in \mathcal{F}(G)\right\}$ ) can be formulated as a matroid parity problem in a certain co-graphic matroid, hence it can be solved in polynomial time. This was observed by Furst et al. [?] who gave a $O\left(e n^{2} \log ^{6} n\right)$ time algorithm based on results from [?]. With the help of this reduction one can obtain Theorem ?? from Lovász's characterization of the maximum size matroid-matching in a linear matroid [?].

A similar reduction, where the matroid is the dual of the sum of $k$ graphic matroids, seems to work in the more general case of Theorem ??, too. However, from algorithmic point of view, such a reduction is not satisfactory, since it is not known how to represent the matroid in question. This difficulty can be handled by a useful observation of S. Iwata [?] which reduces the matroid parity problem on the sum of the $k$ matroids to an instance of the matroid matching problem on the direct sum of the $k$ matroids. It would be interesting to develop a more efficient algorithm for this problem as well as for the problem of Theorem ??.

\section{Acknowledgements}

BRICS (Basic Research in Computer Science) is a Centre of the Danish National Research Foundation. The first two authors were supported in part by the Hungarian National Foundation for Scientific Research grant OTKA T029772.

\section{References}

[1] I. Anderson, Perfect matchings of a graph, J. Combin. Theory Ser. B, 10 (1971), 183-186.

[2] O. Chevalier, F. Jaeger, C. Payan and N.H. Xuong, Odd rooted orientations and upperembeddable graphs, Annals of Discrete Mathematics 17, (1983) 177-181.

[3] J. Edmonds, Edge-disjoint branchings, in: R. Rustin (Ed.), Combinatorial Algorithms, Academic Press, (1973) 91-96.

[4] A. Frank, Orientations of graphs and submodular flows, Congr. Numer. 113 (1996), 111142.

[5] A. Frank, Z. Király, Parity constrained k-edge-connected orientations, Proc. IPCO'99, Graz (G.Cornuéjols, R.E.Burkard and G.Woeginger eds.), LNCS 1610, Springer, 191-201, 1999.

[6] M.L. Furst, J.L. Gross, and L.A. McGeoch, Finding a maximum genus graph imbedding, J. of the ACM, Vol. 35, No. 3, July 1988, 523-534.

[7] H.N. Gabow, M. Stallmann, Efficient algorithms for graphic matroid intersection and parity, in Proc. 12th ICALP, Lecture Notes in Computer Science, vol. 194, Springer-Verlag, New York, 1985, pp. 210-220. 
[8] S. Iwata, personal communication, 1998.

[9] M. Jungerman, A characterization of upper embeddable graphs, Trans. Amer. Math. Soc. 241 (1978), 401-406.

[10] L. Lovász, Selecting independent lines from a family of lines in a space, Acta Sci. Univ. Szeged 42, 1980, 121-131.

[11] C. St. J. A. Nash-Williams, Edge-disjoint spanning trees of finite graphs, J. London Math. Soc. 36 (1961), 445-450.

[12] L. Nebeský, A new characterization of the maximum genus of a graph, Czechoslovak Mathematical Journal, 31 (106) 1981, 604-613.

[13] E.A. Nordhaus, B.M. Stewart and A.T. White, On the maximum genus of a graph, J. Combinatorial Theory B 11 (1971), 258-267.

[14] R.D. Ringeisen, Survey of results on the maximum genus of a graph, J. Graph Theory 3 (1979), 1-13.

[15] N.H. Xuong, How to determine the maximum genus of a graph, J. Combin. Theory Ser. B 26 (1979), 217-225. 